\title{
Interprofessional Collaboration: A Qualitative Study of Non-Physician Perspectives on Resident Competency
}

\author{
Mariposa Garth, BSN ${ }^{7}$, Alexandra Millet, BA ${ }^{7}$, Emily Shearer, MPP, MSc ${ }^{7}$, Sara Stafford, MSN ${ }^{1,2}$, \\ Sylvia Bereknyei Merrell, DrPH, $\mathrm{MS}^{7}$, Janine Bruce, $\mathrm{DrPH}, \mathrm{MPH}^{7}$, Erika Schillinger, $\mathrm{MD}^{1,2}$, \\ Alistair Aaronson, MD, FACP ${ }^{1,2}$, and David Svec, MD, MBA ${ }^{1,2}$
}

'Stanford University School of Medicine, Stanford, CA, USA; ${ }^{2}$ Stanford Health Care, Palo Alto, CA, USA.

BACKGROUND: The Association of American Medical Colleges (AAMC) includes the ability to collaborate in an interprofessional team as a core professional activity that trainees should be able to complete on day 1 of residency (Med Sci Educ. 26:797-800, ${ }^{2016}$ ). The training that medical students require in order to achieve this competency, however, is not well established (Med Sci Educ. 26:457$61,{ }^{2016}$ ), and few studies have examined non-physician healthcare professionals' perspectives regarding resident physicians' interprofessional skills.

OBJECTIVE: This study aims to describe non-physicians' views on barriers to collaboration with physicians, as well as factors that contribute to good collaborative relationships.

PARTICIPANTS: Nurses, social workers, case managers, dietitians, rehabilitation therapists, and pharmacists at one academic medical center, largely working in the inpatient setting.

APPROACH: A qualitative study design was employed. Data were collected from individual interviews and focus groups comprising non-physician healthcare professionals. KEY RESULTS: Knowledge gaps identified as impeding interprofessional collaboration included inadequate understanding of current roles, potential roles, and processes for non-physician healthcare professionals. Specific physician behaviors that were identified as contributing to good collaborative relationships included mutual support such as backing up other team members and prioritizing multidisciplinary rounds, and communication including keeping team members informed, asking for their input, physicians explaining their rationale, and practicing joint problem-solving with non-physicians.

CONCLUSIONS: Discussion of how physician trainees can best learn to collaborate as members of an interprofessional team must include non-physician perspectives. Training designed to provide medical students and residents with a better understanding of non-physician roles and to enhance mutual support and communication skills may be critical in achieving the AAMC's goals of making physicians effective members of interprofessional teams, and thus improving patient-centered care. We hope that medical educators will include these areas

Electronic supplementary material The online version of this article (https://doi.org/10.1007/s11606-017-4238-0) contains supplementary material, which is available to authorized users.

Received April 22, 2017

Revised August 30, 2017

Accepted November 9, 2017

Published online December 4, 2017 identified as important by non-physicians in targeted team training for their learners.

KEY WORDS: communication skills; medical education; nursing; qualitative research; shared decision-making; interprofessional collaboration; interprofessional education. J Gen Intern Med 33(4):487-92

DOI: $10.1007 / \mathrm{s} 11606-017-4238-0$

(c) Society of General Internal Medicine 2017

\section{INTRODUCTION}

Interprofessional collaboration occurs when health providers from different professional backgrounds work together with patients, families, caregivers, and communities to deliver the highest-quality care. ${ }^{1}$ Recognizing the centrality of such teamwork in patient-centered care, the Association of American Medical Colleges (AAMC) included the ability to collaborate as a member of an interprofessional team as one of its 13 newly developed Core Entrustable Professional Activities (EPAs) for Entering Residency. The AAMC specifies eight key functions medical residents must be able to perform on day 1 of residency (Table 1).

The AAMC's recognition of interprofessional teamwork as a core clinical skill has been echoed by the World Health Organization, which identifies a healthcare workforce capable of collaborative practice as necessary for addressing the unmet needs of fragmented healthcare systems. ${ }^{1}$ Team training has been shown to improve clinical care, ${ }^{3}$ and residency directors in the US have ranked incoming interns' ability to communicate and work effectively in teams as a high-priority skill necessary for entering residency. ${ }^{4}$

Despite these calls to action, current evidence suggests postgraduate trainees are not yet achieving the AAMC's core competencies for interprofessional collaboration. ${ }^{5}$ In a study published in this journal, Zabar and colleagues noted that fewer than half of residents performed well on an objective structured clinical exam (OSCE) of core competencies for interprofessional collaboration. ${ }^{6}$ Moreover, the medical student training necessary to develop these skills is not well defined. $^{7}$

There exists extensive teamwork literature to aid in this discussion. TeamSTEPPS is an evidence-based framework used for team training to improve performance across the 
Table 1 EPA 9: Collaborate as a Member of an Interprofessional Team

1. Identify team members' roles and the responsibilities associated with each role

2. Establish and maintain a climate of mutual respect, dignity, integrity, and trust

3. Communicate with respect for and appreciation of team members and include them in all relevant information exchange

4. Use attentive listening skills when communicating with team members

5. Adjust communication content and style to align with team-member communication needs

6. Understand one's own roles and personal limits as an individual provider and seek help from the other members of the team to optimize health care delivery

7. Help team members in need

8. Prioritize team needs over personal needs in order to optimize delivery of care ${ }^{2}$

healthcare environment. Core components of this model include leadership, situation monitoring, mutual support, and communication. ${ }^{8}$ We use TeamSTEPPS as a framework for understanding the areas of improvement described by nonphysician healthcare providers.

This study seeks to broaden discussion around physician skills in interprofessional collaboration by describing nonphysicians' views on this matter in an inpatient setting. By understanding these views, we hope medical educators and residency program directors will be better positioned to target interventions to improve team training.

\section{METHODS}

\section{Study Design and Oversight}

As part of developing an interprofessional education curriculum, we conducted a qualitative study using focus groups and semi-structured interviews to examine non-physician perspectives on residents' competency in interprofessional collaboration. We received an exemption from institutional review board review, as this project was a quality improvement project, not meeting the federal regulatory definition of research.

\section{Setting}

This study was conducted at Stanford Hospital and Clinics, a 444-bed tertiary care center in northern California. Medical teams are staffed by one attending, one resident, two interns, and 1-2 medical students. Since 2014, attending physicians have participated in daily multidisciplinary rounds where they discuss care and discharge plans for patients with the team's dedicated case manager, as well as a dietitian, nurse, rehab therapist, pharmacist, and social worker. The attending visits all nursing units at a specified time to discuss the care plan.

\section{Data Collection}

Data were collected in two phases. The first phase included focus groups to examine experiences collaborating with physicians. Focus groups were followed by interviews with a subset of focus group participants for an in-depth discussion of focus group themes. Participants included members of the interprofessional healthcare team working in adult medicine whose professions were represented at inpatient multidisciplinary rounds. All focus groups were segregated by professional role to facilitate distinguishing profession-specific results from those that were true across multiple professions. Recruitment was via word of mouth, email, flyers, and inperson recruitment at an established professional leadership event. We purposefully sampled ${ }^{9}$ from focus group participants for individual interviews in order to clarify focus group themes and to ensure we were not misinterpreting discussions. We recruited by word of mouth when we lacked sufficient focus group participants to participate in on-camera interviews. Participants were incentivized with $\$ 15$ gift cards and lunch for focus groups, and \$50 gift cards for individual filmed interviews. Participants understood that they were engaging in research to guide the design of an interprofessional education curriculum for Stanford Medical School. They also understood that individual filmed interviews would be included in this curriculum.

Focus groups were conducted by MG; individual interviews were conducted by either MG or SS. MG is a medical student who previously worked as a registered nurse. SS is a registered nurse who currently works at Stanford Hospital. A semistructured focus group guide was developed, informed by Stutsky and Laschinger's work on conceptual frameworks for interprofessional collaboration ${ }^{10}$ and piloted prior to the study (see Appendix 1 for full interview guide). Individual interview questions were drafted based on preliminary focus group theme analysis. Focus groups were audio- and videorecorded using a laptop, and individual interviews were professionally filmed. Focus group discussions and interviews were then professionally transcribed. The qualitative research software Dedoose ${ }^{11}$ was used to organize data for analysis.

\section{Data Analysis}

We analyzed the data in three steps: 1) preliminary thematic analysis of profession-specific focus group findings; 2) member checks on profession-specific focus group findings via individual interviews exploring themes from focus groups; and 3) second-pass coding and thematic analysis of focus group transcripts combined with thematic analysis from individual interviews to determine findings consistent across multiple professions. The codebook was developed from ideas that emerged from the data, with additional codes based on Stutsky and Laschinger's framework. ${ }^{10}$ We performed a phenomenological qualitative inquiry and used coding to identify clusters of meaning. ${ }^{12}$ Due to the large volume of data, and concern with conducting a rigorous inquiry, we performed an inter-rater reliability test to ensure that the coding process aligned between the coders and was true to the research question. ${ }^{13}$ As part of the post-positivist phenomenological 
approach, the researcher understands that they cannot fully know the truth, but having multiple perspectives and rigorous checks along the analytic process ensures that participants' voices are represented more accurately. ${ }^{14}$

A sample size of 2-3 focus groups captures $80 \%$ of topic themes in a relatively homogeneous population. ${ }^{15}$ Given the heterogeneity of our population, including different types of healthcare professionals, we conducted seven focus groups and used data triangulation with individual interviews to ensure thematic saturation. ${ }^{9}$ Individual interview questions were designed to encourage discussion of themes that arose in focus groups, serving as a form of member-checking ${ }^{16}$ of focus group themes. Subsequent analysis compared themes across professions using both focus group transcripts and individual interviews.

\section{RESULTS}

Our seven focus groups included a total of 42 participants, with pharmacists, social workers, case managers, dietitians, nurses, and rehabilitation therapists (speech language pathologists, occupational therapists, physical therapists) participating. Focus groups varied in size from five to seven participants, with two dietitian focus groups due to their high interest in participation. Focus group duration ranged from 30 to $55 \mathrm{~min}$. Most participants came from inpatient settings; however, some professions (pharmacy, social work, dietetics) had at least one person participating who worked in an outpatient setting (see Table 2 for a description of participants). Pooled Cohen's $\mathrm{k}$ for inter-rater reliability for focus group transcripts resulted in a $\mathrm{K}$ of 0.72 . Responses were divided into two domains: knowledge gaps and important components of collaborative relationships (Table 3).

\section{Domain 1: Barriers to collaboration}

Theme 1: Knowledge Gaps. Lack of knowledge of roles led non-physicians to feel underutilized, undervalued and misunderstood by physicians. As one dietitian pointed out: "I think it's important in any working situation that you know the people that you're working with. It just shows a level of respect for how you contribute to the team and what you do." A full list of profession-specific misconceptions can be found in Appendix 2.

Table 2 Participants by Profession and Type of Participation

\begin{tabular}{llll}
\hline \hline Profession & $\begin{array}{l}\text { Focus } \\
\text { groups } \\
(\boldsymbol{n}=\mathbf{4 2})\end{array}$ & $\begin{array}{l}\text { Individual } \\
\text { interview } \\
(\boldsymbol{n}=\mathbf{1 4})\end{array}$ & $\begin{array}{l}\text { Total } \\
\text { discrete } \\
\text { participants } \\
(\boldsymbol{n}=\mathbf{4 6 )}\end{array}$ \\
\hline $\begin{array}{l}\text { Dietitian } \\
\begin{array}{l}\text { Rehab } \\
\text { therapist }\end{array}\end{array}$ & $12(29)$ & $3(21)$ & $12(26)$ \\
$\begin{array}{l}\text { Pharmacist } \\
\text { Nurse }\end{array}$ & $7(14)$ & $4(29)$ & $7(15)$ \\
$\begin{array}{l}\text { Social worker } \\
\text { Case manager }\end{array}$ & $6(17)$ & $2(14)$ & $8(17)$ \\
\hline
\end{tabular}

Table 3 Barriers to Collaboration and Important Components of Collaborative Relationships from the Perspective of Non-Physician Healthcare Professionals

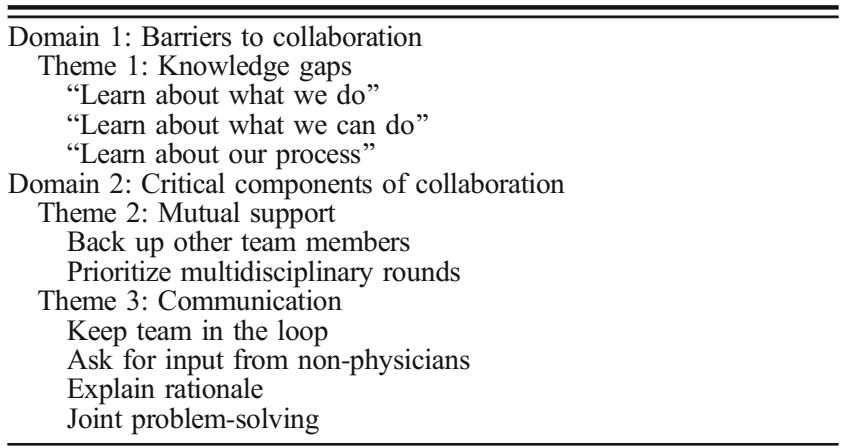

Sub-Theme 1: "Learn About What We Do". Some physicians do not adequately understand the roles of their non-physician colleagues: 'I've seen medical students say, 'Oh, can you just talk to them about low sodium'... I sometimes feel it's just so downgraded to just like, 'just go and talk to them. Give them a sheet of paper.' That's not the way I wanted it" (Dietitian). Many non-physician participants felt that the complexity and nuances of their roles were poorly understood by physicians.

Sub-Theme 2: "Learn About What We Can Do". Social workers, among others, described being unable to work to the full extent of their license because physicians were unaware of the full range of tasks they were qualified to complete.

"We arrange family meetings and we do a lot of processing with families and caregivers and I don't think the medical team sometimes acknowledges how much we can be part of that discussion, especially end-of-life discussions, advance care planning discussions." (Social worker)

Participants often relied on physicians to make them aware of patients who required specialized interventions. Thus lack of physician knowledge was a barrier to their colleagues fulfilling these parts of their roles.

Sub-Theme 3: "Learn About Our Process". Participants described feeling misunderstood by physicians who lacked an understanding of the constraints within which nonphysicians operate:

"We're constantly being told that as a pharmacy department, we need to be optimizing... So, I think that's something we'd like to keep in the back of your head. If we're saying, 'No, this med is too expensive.' We're not saying it because we don't care about the patient. It's just the balance of cost." (Pharmacist) 
Participants expressed a desire for physicians to understand that they were not uncaring, but rather beholden to institutional and logistical constraints.

\section{Domain 2: Critical Components of Collaboration}

Participants identified elements they believed contribute to good collaborative relationships. Much of their discussion revolved around physician behaviors that were seen as improving interprofessional collaboration.

Theme 2: Mutual Support. Participants described physician actions that supported other professional roles. These included agreement between physicians and non-physicians regarding interventions when discussing the plan of care with patients, either when together or individually, and prioritizing teamfocused activities like multidisciplinary rounds.

Sub-Theme 1: Back Up Other Team Members. Participants described feeling that their efforts were wasted when physicians contradicted them on priorities in front of a patient. "Our big issue was trying to control the blood sugar, and so we were working so hard to keep it within certain parameters, and then another doctor walked in was like, 'Oh, we're more concerned about [another area]; you can eat whatever you want'" (Nurse). Part of the feeling of wasted effort arose from the sense that patients give more credibility to physician recommendations than those of non-physician team members. They viewed this authority in the patients' eyes as something that could derail their own interventions if physicians did not express consistent priorities. They also viewed this patient-perceived authority as potentially reinforcing their professional interventions if physicians used it to actively support them: "The physician has a lot of power and a lot of presence, and just by saying, 'do something,' it holds more weight than the lowly dietitian seems to" (Dietitian). Physicians can demonstrate support for non-physician colleagues by using their perceived position of authority to reinforce team priorities.

Sub-Theme 2: Prioritize Multidisciplinary Rounds. Most participants felt that multidisciplinary rounds were crucial for exchanging information within the healthcare team. A case manager described rounds as crucial to her workflow, but that physicians did not always understand them as such: "I often hear [physicians] say, 'I don't get anything out of rounds'... it's not all about what they get out of it; it's about what they offer to enable us to work to get their patient ready when they need to go" (Case manager). Though physicians may not find rounds as useful, participants viewed physician presence and participation at rounds as an expectation for the overall efficiency of the team: "[Multidisciplinary rounds are] the most effective since they are in line of sight, direct communication of the mouth, versus pages here, emails there, back and forth" (Rehab therapist). Physician participation in the face-to-face exchange of information at multidisciplinary rounds is key to non-physician workflow.

Theme 3: Communication. Participants described timely communication from physicians as essential for fulfilling their job duties and providing the best possible patient care. Inclusion of non-physicians in joint problem-solving, both by asking for input and by explaining rationale, were important to collaboration.

Sub-Theme 1: Keep Team in the Loop. Participants noted that they needed physicians to "keep them in the loop" (informed), as many of their job duties required them to know the plan of care. Case managers described needing an estimated discharge date from physicians to prioritize their own work: "Tell us what [estimated discharge date] you're aiming for, because we prioritize our whole day based on that prediction" (Case manager). Pharmacists described needing to know about changes in a patient's condition for accurate and timely adjustment of a patient's medications. "A lot of times [physicians] don't have enough time to put in a note for something that just happened. So, letting us know would be really helpful and help us tailor the dose or just know what's going on" (Pharmacist). Direct communication around patient status and any changes to the plan of care enabled participants to prioritize and to actively contribute to care, and minimized missing key updates and time spent searching through patient charts.

Sub-Theme 2: Ask for Input from Non-Physicians. Participants noted that having physicians seek their input was important for patient care and for them to feel valued by physicians. Physicians soliciting their input meant recognition of their experience and showed they were "all working towards the success of the patient" (Rehab therapist). Responding to the question of how she knows physicians value her, one nurse immediately responded: "by [physicians] asking for [my] input." As one social worker put it, "You don't feel respected if your team is either not giving you the chance to speak your voice or provide your input... or you do and they don't even listen or take it into account". Physicians actively seeking and incorporating input from nonphysicians signals to them that they are valued members of the interprofessional team.

Sub-Theme 3: Explain Rationale. Understanding the rationale behind physician recommendations helps nonphysicians direct their interventions, resolve team conflicts, further their own professional development, and contribute meaningfully to patient care. Dietitians needed to know physicians' rationale for consulting them so that they could target their interventions. For nurses, understanding rationale was 
vital, because they found themselves in the position of needing to explain interventions to patients - a difficult task without understanding the reasons behind physician orders.

Nurses and social workers described understanding physician rationale as a way of mediating disagreement. One nurse described how explaining the reasons for a decision could help resolve conflicts between physicians and nurses: "If I know the rationale of why you're saying no... then I can support you in your decision, and tell the patient 'this is why" (Nurse). For other professionals, understanding physician rationale was key to their own development and learning process. "Having those conversations and understanding why different disciplines recommend this, or why the doctors aren't going to follow our care... That helps us learn and grow too as individuals" (Dietitian). Physicians sharing their rationale with the rest of the team may promote collaboration and foster individual growth.

Sub-Theme 4: Joint Problem-Solving. Apart from pharmacists, all focus groups discussed wanting to be more included in joint problem-solving, with the opportunity to have their professional opinion impact patient care. Many professionals described wanting to be more included in the care plan: "We want to be an integral part of each piece... one of the big challenging things is when you're at rounds and you make a statement, you have an idea, and it's like crickets, and people just don't even realize you're around" (Dietitian). Feeling that physicians listened to their input and engaged them in shared decision-making directly contributed to how valued and respected they felt in the healthcare team.

\section{DISCUSSION}

This study contributes to an extensive body of literature on interprofessional collaboration by providing a crossprofessional assessment of collaboration with physicians, and by identifying specific behaviors non-physician professionals commonly identified as either promoting or impeding collaboration. The healthcare professionals interviewed in this work identified mutual support and communication skills as key to successful collaborative relationships with physicians. These align with components $3,4,5$, and 7 of the AAMC's EPA 9: Collaborate as a Member of an Interprofessional Team (Table 1). Mutual support and communication are also two of the four competencies included in the teamSTEPPS program, along with team leadership and situation monitoring. ${ }^{17} \mathrm{Ac}-$ cording to Marlow et al., healthcare team training most frequently focuses on communication, with $84.8 \%$ of healthcare team training including this competency; mutual support is included in only $22.8 \% .^{18}$ Our results suggest that healthcare team training for physicians may benefit from an increased focus on mutual support.
Pharmacists were outliers in many ways. Among participating professions, pharmacists endorsed much stronger collaborative relationships with physicians: "I think we probably already have a collaborative relationship with the medical team" (Pharmacist). They also did not mention feeling left out of decision-making. Several pharmacists had taken classes with medical students during their training, which may have helped develop this relationship early. In addition, physicians on our team noted that in their experience, during discussions, pharmacists generally present recommendations in the context of journal evidence. Other interdisciplinary fields, while also having a body of evidence to drive decision-making, generally present recommendations without reference to journal evidence. This may contribute to a sense of shared language and decision-making processes between pharmacists and physicians. Future research into how these unique relationships are developed may provide insight into ways to improve other team member relationships.

Mutual respect implies recognition of how the contributions of various professionals on the team complement one another, and an understanding of their interdependence. ${ }^{19}$ Problems in interprofessional collaboration can arise from insufficient understanding of roles and responsibilities, which can in turn adversely affect patient care. ${ }^{20}$ Our work supports the notion that improved understanding of roles is an important component of interprofessional collaboration and can help physicians communicate respect to their non-physician colleagues. This also aligns with EPA 9 components 1 and 6 (Table 1). Medical educators and residency program directors may improve interprofessional collaboration through curricula or training that specifically supports improved role understanding.

\section{Limitations}

Most participants in this study worked in inpatient acute care settings; as such, the results are heavily biased towards barriers to collaboration and suggestions for improvement in this setting. Future research should investigate barriers in other healthcare settings.

Because the primary goal of this research was to drive the creation of an online curriculum, we video-recorded interviews. This likely introduced some social desirability bias ${ }^{21}$ in the study. To ameliorate this, only individual interviews were professionally recorded. Focus groups were recorded from a laptop; this footage was not made public. We felt that the benefits of creating a curriculum with the actual professionals represented (rather than using actors) outweighed the drawbacks of social desirability bias.

Discussions usually centered on "physicians," without distinguishing between medical students, residents, and attendings. This makes it difficult to determine the level of training and practice for which participants' suggestions and observations are most relevant. Questions were usually phrased as regarding "new physicians," and participants understood that the curriculum was for medical students and 
potentially incoming interns. Although it is difficult to parse exactly to whom their suggestions are targeted, we hoped that relaying these concerns to medical students would be an appropriate way to lay the groundwork for better interprofessional collaboration among future medical professionals. Future research on interprofessional skills at different levels of physician training and practice would be valuable.

We encountered particular difficulty recruiting nurses, due to their unpredictable break schedules and because their direct management was divided across multiple hospital units. We chose to recruit at a leadership event in hopes of encountering nurses from multiple areas. We also reduced the nursing focus group duration to $30 \mathrm{~min}$ to accommodate those who wanted to attend on their shorter lunch break. Although this increased the number of participants, these decisions likely skewed nurse representation and may have reduced the depth and breadth of information represented in the nursing focus group.

\section{CONCLUSION}

Discussion of how physician trainees can best learn to collaborate as members of an interprofessional team must include non-physician perspectives. Team training for medical students and residents that emphasizes improved understanding of non-physician roles and focuses on the specific skills of mutual support and communication may be important in better preparing students to practice as members of interprofessional teams, ultimately improving patient-centered care. These elements are all key components of both the AAMC's EPA 9 and the TeamSTEPPS framework. We hope that medical educators will include these areas identified as important by nonphysicians when designing team training for medical students and residents.

Acknowledgements: We would like to thank participating healthcare professionals at Stanford Health Care, as well as R.J. Sanchez and Alison Brauneis, who were integral to curriculum development. We are grateful for a grant from the Stanford Office of the Vice Provost for Teaching and Learning, and support from Dean Prober and the Medical Scholars Program of the Stanford School of Medicine. This research was presented at the Western Group on Educational Affairs Regional Meeting and the Stanford Innovations in Medical Education Conference.

Corresponding Author: Mariposa Garth, BSN; Stanford University School of Medicine, Stanford, CA, USA (e-mail: mgarthp@stanford. edu).

Compliance with Ethical Standards:

Conflict of Interest: All authors declare that they have no conflict of interest.

\section{REFERENCES}

1. Gilbert JH, Yan J, Hoffman SJ. A WHO report: framework for action on interprofessional education and collaborative practice. J Allied Health. 2010;39(Suppl 1):196-7.

2. Association of American Medical Colleges. Core Entrustable Professional Activities for Entering Residency: Curriculum Developer's Guide. Washington, DC: Association of American Medical Colleges; 2014.

3. Capella J, Smith S, Philp A, et al. Teamwork training improves the clinical care of trauma patients. J Surg Educ. 2010;67(6):439-43.

4. Angus S, Vu TR, Halvorsen AJ, et al. What skills should new internal medicine interns have in July? A national survey of internal medicine residency program directors. Acad Med. 2014;89(3):432-5.

5. Lomis KD, Ryan MS, Amiel JM, Cocks PM, Uthman MO, Esposito KF. Core entrustable professional activities for entering residency pilot group update: considerations for medical science educators. Med Sci Educ. 2016;26(4):797-800.

6. Zabar S, Adams J, Kurland S, et al. Charting a key competency domain: understanding resident physician interprofessional collaboration (IPC) skills. J Gen Intern Med. 2016;31(8):846-53.

7. Brown DR, Gillespie CC, Warren JB. EPA 9-collaborate as a member of an interprofessional team: a short communication from the AAMC Core EPAs for entering residency pilot schools. Med Sci Educ. 2016;26(3):45761.

8. King HB, Battles J, Baker DP, et al. TeamSTEPPS: team strategies and tools to enhance performance and patient safety. In: Henriksen K, Battles JB, Keyes MA, Grady ML, eds. Advances in Patient Safety: New Directions and Alternative Approaches (Vol 3: Performance and Tools). Rockville: Agency for Healthcare Research and Quality (US); 2008.

9. Carter N, Bryant-Lukosius D, DiCenso A, Blythe J, Neville AJ. The use of triangulation in qualitative research. Oncol Nurs Forum. 2014 Sep;41(5):545-7.

10. Stutsky BJ, Spence Laschinger, Heather K. Development and testing of a conceptual framework for interprofessional collaborative practice. Health Interprof Pract. 2014;2(2):eP1066.

11. Dedoose Version 7.5.9. Web application for managing, analyzing, and presenting qualitative and mixed method research data (2016). Los Angeles: SocioCultural Research Consultants, LLC (www.dedoose.com); 2016.

12. Creswell JW, Poth CN. Qualitative inquiry and research design: Choosing among five approaches. Thousand Oaks, CA: Sage Publications; 2017.

13. Campbell JL, Quincy C, Osserman J, Pedersen OK. Coding in-depth semistructured interviews: problems of unitization and intercoder reliability and agreement. Sociol Methods Res. 2013.

14. Clark AM. The qualitative-quantitative debate: moving from positivism and confrontation to post-positivism and reconciliation. J Adv Nurs. 1998;27(6): 1242-9.

15. Guest G, Namey E, McKenna $\mathbf{K}$. How many focus groups are enough? Building an evidence base for nonprobability sample sizes. Field Methods. 2017;29(1):3-22

16. Krefting $\mathbf{L}$. Rigor in qualitative research: the assessment of trustworthiness. Am J Occup Ther. 1991;45(3):214-22.

17. Guimond ME, Sole ML, Salas E. TeamSTEPPS. Am J Nurs. 2009;109(11):66-8.

18. Marlow SL, Hughes AM, Sonesh SC, et al. A systematic review of team training in health care: ten questions. Jt Comm J Qual Patient Saf. 2017;43(4): 197-204.

19. San Martin-Rodriguez L, Beaulieu MD, D'Amour D, Ferrada-Videla M. The determinants of successful collaboration: a review of theoretical and empirical studies. J Interprof Care. 2005; 19(SUPPL. 1): 132-47.

20. Zwarenstein M, Goldman J, Reeves S. Interprofessional collaboration: effects of practice-based interventions on professional practice and healthcare outcomes. Cochrane Database Syst Rev. 2009 (3).

21. Nederhof AJ. Methods of coping with social desirability bias: a review. Eur J Soc Psychol. 1985;15(3):263-80. 